Regarding the individual colleges the inspector made the following statements:

Davenport College of Chiropractic.-"Their alleged "chemical laboratory' consisted of a table about 18 inches square, on which were six dusty bottles and three dirty test tubes. There were no records of work done by the students or of attendance at lectures or clinics."

Universal Chiropractic College.- "They are not in any way equipped to give the character of instruction that would make their graduates safe advisers to the sick."

Palmer School of Chiropractic.- "They pretend to give a course in obstetrics with no practical experience. A person who assumed to practice on information gained from this course alone would be dangerously incompetent.

"Some of their professors are exceedingly ignorant. The 'professor' of chemistry alleged he taught the 'Widal test' chemically, but chemicals for even ordinary tests were not in evidence: those in evidence showed no marks of use, most of the bottles being still sealed.

"The institution is not physically equipped to turn out safe graduates."

A letter from Dr. J. M. Baldy, president of the Pennsylvania Bureau of Medical Education and Licensure, to whom the manuscript of this article was submitted, states as follows:

"The data quoted from our inspection is correct. I have gone over it myself and have submitted it to the inspector."

It is sufficient to say that the three schools are uniform on the following points: None has a library, a hospital, a laboratory that is worthy of the name, postmortems or capable teachers. Add to this farce students without preliminary education beyond the mere ability to read and write and who "graduated" after a course of only one year in one of these "colleges," and we have an individual who is hopelessly handicapped for life, one who cannot in any sense be considered a safe adviser of the sick.

These reports set forth everything with painful exactness and embarrassing detail. Any patriotic legislator will be justly incensed at the thought of taking the intellectual refuse from these schools who are not pernitted to practice in Iowa, to be used in his own state as the guardians of life and health. They should not be given legal recognition to practice in any state. If they are permitted to practice, it should be only after they have obtained the same professional and preliminary training that is required of physicians, which in Iowa is a high school education and two years of collegiate work plus four years of professional training in a medical school that maintains a standard satisfactory to the licensing board.

Inconsistency of the Antivivisectionist.-Of course there are all sorts of men among physicians and surgeons as in all o.her professions. Abuses and outrages do occur, no doubt. There have been wicked doctors who have abused their trust; and there have been clergymen with whom the virtue of a young lady boarder was not safe; but we need not say for this reason that all surgeons are arch torturers and that all preachers are arch-lechers. Just as you do not need to pass a special law against adultery by ministers of the gospel, but that if you did so you would put an imputation on the character of a large body of earnest, sincere and unselfish men, so you should not pass laws which would put on men in biological research the imputation of bad faith and cruelty. Make the general laws against cruelty to animals as strict and far-reaching as may seem necessary.for the good of the human race; but do not single out the men who are devoting their lives to the search after that knowledge which is for the best good of the race, and make them the special objects of unnecessary restrictive limitations. If experiments on animals must be prohibited let the same law prohibit castration of animals and the dehorning of cattle. If the English law requiring all operations by a scientific man to be done under anesthesia be adopted, then require that operations on the farm be performed in the same way.--Maxwell, Science.

\section{RECTAL AS A SUBSTITUTE FOR VAGINAL EXAMINATIONS. IN LABOR}

RUDOLPH WIESER HOLMES, M.D.

Associate Professor of Obstetrics and Gynecology, Rush Medical College CHICAGO

In spite of the most rigorous regard to the details of asepsis in the preparation of the patient, of the attendant's hands, and of the supplies which may directly or indirectly approach the two, the dangers of puerperal infection have not been reduced to that negligible degree which our profession should attain. Since the days of Carl Braun, various authorities have recog. nized the dangers accompanying vaginal examinations, have strenuously demanded the diminution of their number, and have sought means whereby such curtailment might be possible. All modern authorities are agreed that vaginal examinations, no matter with what conscientious regard to aseptic technic they are conducted, carry with them their risk of sepsis. Today it is generally recognized that abdominal palpation, aided by a proper interpretation of clinical evidences, offers as much, if not more, certitude as to the condition of the woman and progress of labor than do vaginal examinations alone. The elimination of the necessity of entering the vagina, or at least reducing the frequency to the point at which imperative exigency demands it, will be a distinct obstetric advance. In this paper, I would show that rectal examination fulfils this purpose, and that rectal will secure as much definite information, in most particulars, as do vaginal examinations, and without the risks of infection which inevitably accompany the latter.

When ventral fixation was a popular procedure, I found it expedient, in a number of women in labor, to endeavor to reach the cervix by rectal palpation. This was merely applying a method resorted to in gynecologic cases when, as in virgins, it was impracticable or impossible to use the vaginal route. However, this did not suggest the full value of the method in the conduct of labor. Two or three years ago the broader application was grasped from a source not determined. At first, used in an incidental manner, then as a routine, an experience matured which has convinced me that it was an invaluable method of investigation. Now, I am certain that rectal examination, supplementing abdominal palpation, should largely supersede vaginal touch in the conduct of normal labor, and in certain pathologic cases to be hereinafter mentioned; the latter route should be reserved solely for those cases in which the findings are inconclusive, or as a preliminary to operative work through the parturient canal.

Rectal, as a substitute for vaginal examination, was suggested by Kroenig, ${ }^{1}$ Nov. 20, 1893, in a paper read before the Obstetric Society of Leipzig. The method was introduced in his clinic early in August, 1893. The midwives quickly developed a facility in the procedure, so that in the three months covered by his report, 90 per cent. of all labors were conducted by rectal examination alone. He deduced the following conclusions :

1. Midwives could palpate the spines of the ischia, determine the movability of the coccyx, and estimate the degree of resistance of the perineum.

2. They could determine whether the presenting part were in the pelvis or not.

1. Kroenig: Zentralbl. f. Gynäk., March 10, 192! 
3. They could recognize the sutures of the forelying head.

4. They could determine the relationship of the crown of the head to the bisischiatic line.

5. They could determine the dilatation of the os definitely in multiparas. In primiparas, errors were apt to occur, when effacement was completed.

6 . Generally the fontanels were not to be differentiated.

7. The promontory was palpable only when a high grade of pelvic contraction existed.

Independently of Kroenig, Emil Ries, ${ }^{2}$ now of Chicago, iegan an investigation of the possibilities of rectal examination, in January 1894, in the Strassburg clinic, and published his results a few weeks after Kroenig. The possibilities of the method were largely determined by Kroenig through the work of the midwives. Therefore, we have conclusive evidence of its worth as a means for general application. Ries' deductions were fixed largely by the results obtained by his own personal experience. As a consequence, his findings are more valuable; more comprehensive and more utilitarian for the working needs of the medical attendant. He corroborates the teachings of Kroenig, but has determined the far wider range of its applicability than Kroenig concedes for the method. Ries has presented the only extensive monograph on the subject, an opinion based on a very cursory review of the literature. Only one American textbook on obstetrics mentions the utility of rectal examinations, in a paragraph which includes four or five lines. F. Menge ${ }^{3}$ gives an adequate interpretation of the application of rectal examination. He says, "If one combines it [abdominal palpation] with rectal examination, then after a little practice, one is able to judge in every single case all diagnostic and prognostic details with certainty, even if the case comes after the onset of labor." He further recommends that a single vaginal touch is to be undertaken only when the combined external palpation and rectal examination is not clear in every particular.

That the logical position of rectal examination may be properly presented, I shall give the usual factors which must be determined by routine vaginal exploration. These are taught in most recent textbooks, and are the factors which essentially are to be determined by the new method of investigation. At this point I can but mention the necessity of a definite procedure with every woman who presents her case to the obstetric attendant: The physician should make it an invariable rule to study carefully every woman by repeated abdominal and pelvic examination during the months of pregnancy, in order that he may be conversant with her normality or abnormality, so he may properly and intelligently supervise the labor.

\section{FACTORS TO BE DETERMINED}

Vaginal examinations in pregnancy, as we have learned by precept and example, should comprehend the determination of the following facts:

1. The pelvic size, its normality or abnormality.

2. Soft parts: their normality or abnormality: (a) the perineum; $(b)$ the vagina; $(c)$ the cervix and os; $(d)$ recognition of tumors in or about the lower segment - placenta praevia, etc.; $(e)$ the réctum.

2. Ries, Emil: Zentralbl. f. Gynäk., April 28, 1894. 3. Menge F.: Winckel's Handbuch der Geburtshülfe, i, Part 2, p. 1208 .
3. The fetus: presentation and position ; the cephalopelvic adaptability.

Further, in labor additional data must be determined:

4. The fetus: (a) revisional diagnosis of presentation and position, which must be made because many of the deviations from the normal are the products of labor; $(b)$ the station of the presenting part; $(c)$ the fact of compound presentation, as prolapse of tine aim, leg or cord ; $(d)$ fetal deformities.

5. The effacement of the cervix, and dilatation of the os.

6 . The status of the membranes.

Of course, in the event that the woman is first seen with labor in progress, all the foregoing data must be determined at the first examination, and may demand a vaginal examination.

There may be no question of the expediency of combining external palpation with vaginal examination in pregnancy, for then, carefully conducted, in a cleanly manner, the danger is negligible. To control the vaginal findings by rectal touch, in pregnancy, will convince the most skeptical of the desirability and reliability of the latter, and will manifest to him the certitude and conclusiveness of the knowledge obtained by it. The information obtained by these means, and properly recorded for reference in labor, will determine with definiteness the potential possibilities of the safe passage of the child. Therefore, in labor, only the supplemental factors have to be elicited by a pelvic examination - and they may be conclusively fixed by rectal touch.

The Pelvis.-The configuration of the posterior half of the pelvis may be outlined with clearness, in many respects with a precision that is impossible vaginally. The contour of the sacrum, its irregularities and relation to other osseous structures may be determined; the position and movability of the coccyx are evident. The spines of the ischia may be palpated, and their influence on labor may be approximated, as in the case of the ischia themselves. I cannot concur. in Ries' contention that the anterior pelvic half may be palpated as definitely rectally as vaginally. Likewise, I have not convinced myself that the diagnosis of pelvic size is easily feasible rectally, though I do not doubt that some resourceful mind will discover a practical means of determining the conjugata diagonalis, even the vera, rectally. I have not been able to determine the diagonal conjugate, though the cephalopelvic relationship may be judged with a certain precision.

The Soft.Parts.-Generally, the condition of the soft parts may be determined with the same accuracy as by the vaginal examination; the perineum may be studied with clearness, the texture of the vagina and of the cervix may be readily estimated, and the important data appreciated. The progress of the effacement of the cervix may be observed, and the degree of dilatation of the os measured; the clearness of recognition is particularly evident if the cervix is directed posteriorly. It is a clinical fact that effacement of the cervix and dilatation of the os occur synchronously in multiparas. In primiparas, the rule is for effacement to occur; then dilatation follows. Therefore, it is more easy to recognize the degree of dilatation in multiparas. In primiparas, the difficulty of appreciation is due to the fact that the thinned cervix fits snugly over the bulging membranes without a thick ring to designate the os. As Ries has well pointed out, by pal- 
pating the cervix at the height of a contraction, little will be felt; in the interval of contraction, the lessened tension of the membranes will allow an indefinite band to develop which represents the boundary of the os. $i$ le also shows that the degree of dilatation is of relatively slight importance. Its size merely designates, to a certain extent, the progress of labor. An emergency which imperatively demands immediate operative intervention is not influenced by the fact that the os is dilated two or four fingers - a dilatation must be consummated artificially. The employment of this method has not been occasioned in placenta praevia, but, a priori, its recognition by rectal means would be influenced by the same factors which would permit its easy or difficult palpation vaginally. Often, a tumor may be outlined better by rectal examination than vaginal.

The Fetus.-In the majority of cases the diagnosis of presentation and position may be made without difficulty, providing the presenting part be within the pelvic cavity, that is at least engaged; often, it is surprising with what degree of certainty the sutures and fontanels may be palpated. This is especially true after material dilatation has occurred. Unquestionably, the facility with which they may be recognized rectally is directly commensurate to the ease with which they may be differentiated vaginally. We all occasionally encounter women in whom it is well nigh impossible to recognize the landmarks of the fetal head on account of the advanced ossification, or from marked overriding of the bones the result of excessive molding, or a tense caput; or, finally, the pelvic floor may be so tense that it precludes proper digital exploration. In such, when conditions demand certainty of diagnosis, and external palpation is difficult or impossible, the necessity arises of passing the hand into the lower uterine segment, under deep anesthesia, before the position may be accurately fixed. In such cases, the rectal offers no more indefiniteness than the vaginal route. It has not been my fortune to encounter a compound presentation, during the period I have employed the method as a routine, but $I$ am convinced that it will offer almost the same certitude of recognition as will the vaginal examination, especially if the compound presenting part be laterally or posteriorly directed. It has been determined that the maneuvers of Müller and Kcrr may be applied through the combined abdominal and rectal palpation when the vaginal is replaced by the iatter. This is of fundamental importance, in labor, when the woman has a pelvic contraction and it is necessary to determine approximately the cephalopelvic adaptability when a cesarean section is under advisement. The station of the presenting part, whether floating, fixed, engaged or deep in the pelvis, all may be as positively estimated by rectal as vaginal examination. Ries has found that the pulsations of the cord in the vagina may be felt through the rectovaginal septum. Even the still cord may be outlined in the same manner, showing the possibilities in the diagnosis of a prolapsed cord.

The Membranes.-If there is any distention of the amniotic sac by liquor amnii it is possible to determine the fact. In a few instances it has been possible to rupture the membranes by firmly pressing on them through the rectovaginal septum at the height of a contraction. If there be a very scanty amount of forewater, or it is practically absent, one necessarily must be kept in ignorance; but it has happened in a few instances (as in oligohydramnios) that the only way to determine the presence or absence of the amniotic sac is to open the vagina widely with specula and inspect the head to ascertain if it be covered with the chorion.

Nowhere does the efficiency of rectal examination stand out so distinctly as in the control of women who have a relative indication for cesarean section. In such, it is not good obstetrics, usually, to perform the section until by a test of labor it has been positively proved that the disproportion is so material that an intact, living child cannot pass. We all know that the surest prognostic aid in determining the outcome of a cesarean section is the fact that vaginal examinations have or have not been made within a period of two or three days of the operation. It cannot be reiterated too often and emphatically that the contamination produced by one or more vaginal examinations during the labor influences the outcome of a cesarean section more unfavorably than does any other factor except exhaustion or ill-advised attempts at delivery. It is possible to determine the nonengagement of the head by abdominal palpation and rectal examination with absolute positiveness: therefore, it is utterly needless to enter the parturient canal during the period of watchful expectancy.

Rectal examination at or near term is by no means the obstetric limit to the procedure, for it is efficacious at any period of gestation. In threatened abortion, $o \because$ abortion in progress, the rectal touch will furnish many of the data requisite for an intelligent conduct of the case up to the point of operative intervention.

\section{TECHNIC}

The woman should be given an enema before examination. Preferably, she should be on a table, or at least she should lie across the bed in the lithotomy position, with the buttocks quite to the edge, so the gluteal groove will not be in a depression of the bedding. A sterile rubber glove should be employed, for even though the finger enters a contaminated cavity it is not good practice to use a glove which may have infective matter on it. The finger should be anointed with sterile petrolatum or other unguent, and then should be passed through the anus slowly and carefully. At the same time the other fingers should be flexed in order that they may not accidentally enter the vulvar orifice. The various movements of the fingers should be slowly made, that the minimum of discomfort may be caused. Rarely will there be any pain, even if hemorrhoids are present; in fact, the average discomfort is no greater than when a vaginal examination is made. Generally, the rectovaginal septum is so lax that the examining finger may move with great latitude. The only time I found the method positively indefinite was in a woman who had had proctitis, with secondary contraction of the wall.

\section{DANGERS}

Very possioly objections to the method may be developed in the course of a broader experience objections which may be valid in a very circumscribed field, and limited, in all probability, to conditions in the rectum itself. As we now see it, the danger is that the inexpert may allow the fingers or thumb to slip within the introitus during certain manipulations. This will be entirely obviated by keeping them flexed. The method is contraindicated when the termination of the labor is imminent, for fear, if haste is necessary, the attendant might forget to change the glove, or which is 
quite as bad, attempt to wash the glove on his hand. The glove, after use, invariably should be boiled. Preferably, this glove should not be used in the conduct of the labor, even though it has been sterilized by prolonged boiling. In the old days when the method was introduced there was an obvious objection to it, as the examining finger necessarily was fouled, and sterilized with difficulty. Esthetically, also, it was not agreeable to contaminate the finger with fecal matter. Today, with the use of rubber gloves, there can be no such objection.

The basic principles on which this paper is founded were obtained in the course of my personal experience and practice. In substance they form the essentials of my teaching of pelvic examination in the class room. In the obstetric clinical instruction of the students of Rush College, rectal examination is practiced nearly to the exclusion of vaginal examination. Those who have charge of this work are convinced that the students have had no sacrifice of a means of teaching since vaginal examinations were eliminated. On the contrary, with the increased license of examination, the students have had an increased opportunity of pelvic investigation.

Since Dr. N. Sproat Heaney introduced this procedure in the dispensary clinic, the students have enormously augmented their practical knowledge of the phenomena of labor - are infinitely better placed than were the students who were permitted to make a limited number of vaginal examinations in a former period.

\section{CONCLUSIONS}

1. The discovery of the possibilities of rectal examination in labor by Kroenig and Ries is one of the most important contributions to modern obstetric medicine of the last generation.

2. There must be a complete revision of the chapter on pelvic examination in obstetric textbooks. Adequate presentation of the value of rectal examination must be stated. Vaginal examination should be made subordinate to rectal touch.

3. While, at the present time, rectal examination does not give us the means of measuring pelvic size, it is of great value in estimating the factors situated in the posterior half of the bony pelvis.

4. In almost every direction, under appropriate conditions, rectal examination is as definitive as vaginal, if combined with abdominal palpation.

5. The ease or difficulty of rectal examinations are commensurate to the ease or difficulty which encompass the vaginal ones, and are dependent on the same factors.

6. Rectal examination is an utterly innocuous procedure in labor, whereas, vaginal examinations are always potential sources of infection to the woman.

7. Rectal examinations are peculiarly appropriate to those women who are to receive the test of labor in relatively contracted pelves when a cesarean section may possibly be needed.

8. Vaginal examinations should be made in labor only when, for some special reason, the rectal findings are indefinite or inconclusive.

9. The least that can be said of rectal examination is that it permits the conduct of normal labor with the essential elimination of vaginal examinations : a decided advance in the technic of the conduct of labor.

122 South Michigan Avenue.

\section{THE RAT A CARRIER OF A DYSEN- TERIC AMEBA *}

KENNETH M. LYNCH, M.D.

CHARLESTON, S. C.

The purpose of this communication is to record observations incriminating the rat (Mus norvegicus) of a possible new rôle in the dissemination of disease, the details of which go to show that:

1. The rat (Mus norvegicus) suffers from spontaneous amebic dysentery similar to that occurring in man.

2. This apparently occurs in endemic foci in Charleston, S. C.

3. The ameba found in the cases of rat infection here reported are indistinguishable from Endamoeba histolytica.

4. This rat may be infected with Endamoeba histolytica by allowing it to eat human feces containing this parasite.

5. The disease thus produced is practically the same as that occurring in man and from spontaneous infection in rats.

6. This infection, spontaneous and experimental, may be transmitted from the infected to the healthy rat by close association, that is, living, eating and drinking together.

7. The rat is a possible and probable disseminator of dysenteric amebas pathogenic for man.

The details of the study from which these conclusions are drawn are as follows:

During the course of experimental transmission of the Trichomonas intestinalis to rats by feeding them human feces containing these parasites, ${ }^{1}$ a large brown rat was given feces containing Endamoeba histolvtica in addition to the trichomonas. At the end of three weeks the rat was noticed to be getting thin in spite of gcod feeding, and to be sluggish whereas he had been active and vicious. His emaciation became more noticeable in the course of a few days, and at the end of a month he appeared to be very sick and had developed a mucous diarrhea. This passed fecal matter, when examined fresh and warm, contained, in addition to the trichomonas and leukocytes, desquamated epithelium and shreds of intestinal mucosa, and red blood cells, numerous active and resting amebas identical with those in the feces with which he was fed. $H$ is feces as well as that of all the other animals used in this study had been examined for amebas before feeding and none found. His symptoms, sluggishness, dyspnea, turning of the hair, loss of appetite and diarrhea continued until he died on the thirty-second day after feeding.

Postmortem examination revealed a general anemia, acute mesenteric lymphadenitis, late perilobular cirrhosis of the liver with microscopic periportal abscesses in which no cells like amebas were found, jaundice of the liver and kidneys. The condition of the liver was probably due to the extensive infiltration with cysticerci and secondary infection. The following was the condition of the alimentary canal.

Stomach: Considerable mucus formation, otherwise normal.

Small intestine: Distinct acute catarrhal enteritis, fluid mucinous contents, no ulceration, no amebas present but many lamblias.

* Read before the Medical Society of South Carolina, Nov. 15, 1915

1. Lynch, K. M.: The Tricho:ronas as a Parasite of Man and Certain l.ower Anima!s, Tr. Pan-American Med. Cong., 1915. 